\title{
Optimizing the Configuration and Control of a Novel Human-Powered Energy Harvesting System
}

\author{
Vishwa Goudar*, Zhi Ren ${ }^{\dagger}$, Paul Brochu ${ }^{\dagger}$, Qibing Pei ${ }^{\dagger}$ and Miodrag Potkonjak* \\ ${ }^{*}$ Computer Science Department, University of California, Los Angeles \\ ${ }^{\dagger}$ Department of Material Science and Engineering, University of California, Los Angeles
}

\begin{abstract}
As sensor equipped wearable systems enter the mainstream, system longevity and power-efficiency issues hamper large scale and long-term deployment, despite substantial foreseeable benefits. As power and energy efficient design, sampling, processing and communication techniques emerge to counter these issues, researchers are beginning to look on wearable energy harvesting systems as an effective counterpart solution. In this paper, we propose a novel harvesting technology to inconspicuously transduce mechanical energy from human foot-strikes and power low-power wearable systems in a self-sustaining manner. Dielectric Elastomers (DEs) are high-energy density electrostatic transducers that can transduce significant levels of energy from a user while appearing near-transparent to her, if configured and controlled properly. Towards this end, we propose DE-based harvester configuration that capitalizes on properties of human gait to enhance transduction efficiency, and further leverage these properties in an adaptive control algorithm to optimize the net energy produced by the system. We evaluate system performance from detailed analytical and empirical models of DE transduction behavior, and apply our control algorithm to the modeled DEs under experimentally collected foot pressure datasets from multiple subjects. Our evaluations show that the proposed system can achieve up to $120 \mathrm{~mJ}$ per foot-strike, enough to power a variety of low-power wearable devices and systems.
\end{abstract}

\section{INTRODUCTION}

As Wireless Sensor Network (WSN) systems and techniques mature and standardize, sensor integrated wearable systems are opening up new avenues in several fields, spanning medicine, environmental monitoring, participatory sensing, human-computer interaction and entertainment. However, their energy demands continue to limit operating lifetimes and sustain maintenance overhead, especially in the mobile context, as battery energy densities evolve at a significantly lower pace [1]. To alleviate this problem, several optimizations have been offered for wearable system design spanning energy-efficient wearable node configuration [2], communication power optimization [3], energy-efficient compressed sensing [4], powerefficient context-aware sensor subset selection [5] and plugand-play systems that support heterogeneous harvesters [6], to name a few. In the context of low-power wearable systems that involve human locomotion, we propose the use of a leadingedge energy harvester technology to scavenge the energy produced by a user's foot strikes and drive a target wearable system in a self-sustaining manner.

Foot strikes have been estimated to yield significant amounts of energy - a 154-pound person can produce upwards of $7 \mathrm{~W}$ per foot strike [1]. However, the portion of this that can be scavenged is limited by two factors. First, current energy transduction technologies are characterized by energy densities that are insufficient to capture such quantities of energy. Second, state-of-the-art transducer configurations are unable to transduce significant proportions of human energy output without causing discomfort to the user. For example, a hydraulic-amplified piezo-electric based foot-strike energy harvesting system was proposed in [7], capable of producing $675 \mathrm{~mW}$ per foot-strike while adding significant heft to the shoe, thereby altering the user's gait. In contrast, the authors of [8] proposed a piezo-electric based foot-strike energy harvesting system that interfered minimally with the user's gait and was capable of producing $10-20 \mathrm{~mW}$.

Recently, a new class of harvesters have emerged with the promise of superior transduction ability and minimal impact on user comfort. Dielectric Elastomers (DEs) are high energydensity rubber-like materials that possess the ability to behave as energy generators, actuators and sensors. Their ability to yield 5 to 40 times the energy density of piezoelectrics [9] makes them more effective transducers. Their soft rubber-like nature further enables near-transparency of the harvesting subsystem to the user. However, while competing technologies transduce ambient energy by exploiting underlying physical phenomena (e.g. seebeck, photovoltaic and piezoelectric effects), DEs transduce mechanical energy based on an electrostatic principle. Hence, their configuration and control is key to the levels of energy that they may harvest.

In this paper, we propose a DE harvester array configuration for improved transduction efficiency of the mechanical energy from foot pressure. We leverage the users plantar pressure profile to control the design of individual transducers in the array, such that the energy yield is more uniformly high across the foot despite spatial variations in the pressure profile. High efficiency is also achieved via direct transduction of input mechanical energy. Finally, its mechanical simplicity keeps system design and maintenance complexity to a minimum. We also propose a novel adaptive closed-loop DE transduction control paradigm that exploits characteristics of the user's gait to maximize energy output. As electrostatic transducers, DE output is heavily reliant on the control timing during its transduction cycle, which will vary between foot steps owing to variability in the user's gait. To maximize energy output, we propose an adaptive control algorithm that makes use of fool pressure sensors to predict optimal control timing of each harvester in the array, based on statistical characteristics of the user's gait. The sensors are sampled to maximize the net energy harvested under sampling power constraints imposed by the power demands of the target system.

We validate our proposed transducer configuration by experimentally characterizing and modeling its behavior in detail. The resulting models, in conjunction with our proposed adaptive control algorithm, are applied to experimentally collected datasets of foot pressure of multiple users to demonstrate that our DE based foot-strike energy harvesting system can yield 
upwards of $120 \mathrm{~mJ}$ per foot-strike, thereby producing enough energy to independently power a number of wearable platforms while the user walks [10], [11], [12].

\section{RELATED WORK}

Energy harvesting mechanisms may be classified into ambient radiation, electromagnetic, electrostatic and piezoelectric transduction, among others [13]. Whereas solar radiation transducers offer the most mature and impactful solutions, in the context of mobile devices and wearable systems, their placement requirements and the sporadic availability of bright light hampers adoptability. Electromagnetic generators are most effective and widely popular at the macro-scale, however, they are obtrusive and difficult to miniaturize for the targeted application domain. Although recently, kinetic energy harvesting with wearable electromagnetic generators has been proposed. RF radiation, thermoelectric and conventional air-gap electrostatic harvesters are limited in their output capacity ( $\mu \mathrm{W}$ to low $\mathrm{mW}$ range [1]) with electrostatic harvesters further hindered by their composition of moving parts. Finally, piezo-electrics have higher energy densities as compared to electrostatic and electromagnetic transducers, and are also well suited for harvesting the vibrational forces commonly experienced by human bodies [13]. However, as we shall see in section III-A, although DEs are electrostatic transducers, their superior material properties distinguish them from conventional transducers in this class, making them a promising new candidate.

In the wearable systems and body area networks community, it is generally agreed upon that energy harvesting poses an important challenge and opportunity for self-sustenance [14]. Aside from body heat transfer via the skin and vibration from foot strikes, proposed human-powered transduction alternatives include movement of knee joints, inertia from backpacks and change in blood pressure. A wide array of human motion has been found suitable for exploitation including cranking, shaking, pumping, pulling as well as the isometric forces of squeezing and pushing [1], [13]. However, human gait offers easy pickings as the most innocuous source of human power for transduction, resulting in a long trail of harvesting designs and related patents going back to the mid 1920's [15]. Two such designs were described in section I.

DEs are a relatively new entrant to the class of miniaturized generators. An excellent survey of the material properties relevant to its transduction mechanism, various proposed transducer configurations, capabilities in comparison to other common transducers, recent applications, and, operational boundaries and lifetime issues are detailed in [9]. However, due to its basis in electrostatic transduction, maximizing the output of a DE generator, strongly relies on the transducer configuration and control methodology. We also note that DEs require charging at high voltage so they may achieve their output potential; However self-priming circuits have been proposed that use an inverse charge pump to convert some of the DE voltage boost into charge, incrementally increasing the source voltage from $10 \mathrm{~V}$ to the $\mathrm{kV}$ range [16]. Finally, adaptive control of DEs has been proposed in the context of actuation [17], however to the best of our knowledge, we are the first to propose an energy-maximizing adaptive control technique for DE generators.

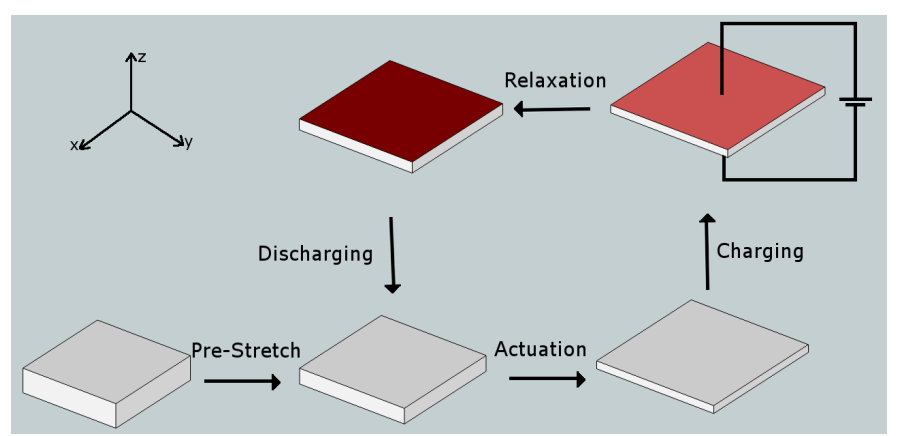

Fig. 1: Dielectric elastomer generator transduction cycle.

\section{PRELIMINARIES}

\section{A. Dielectric Elastomer Generators}

DEs are deformable yet incompressible insulating polymer films with high electric permittivity $\left(\varepsilon_{r}\right)$ and relatively low mechanical and electrical losses. This leads naturally to their use as variable capacitors capable of direct transduction of mechanical to electrical energy. Further, their relatively high elastic energy density ( 5 to 40 times that of piezoelectrics) makes them extremely productive as transducers as they can store more energy when deformed for the same amount (mass and volume) of transducer material [9]. At the same time, they are quite soft compared to piezoelectrics, making them less intrusive and more acceptable for human use.

The operation of a DE as a generator occurs in 4 stages (Fig. 1). The DE is first stretched by the mechanical pressure applied to it in the actuation stage, thereby increasing its capacitance. In the charging stage, electrical charge is be added to the elastomer's surface at a fixed voltage $V$, generating a charge proportional to the capacitance. Throughout the transduction stage that follows, the amount of charge $Q$ on the DE is held constant. Here, as the applied mechanical pressure reduces to zero, the elastic forces in the DE relax and are transduced into electrostatic force. As the DE relaxes, its capacitance decreases leading to an increase in its voltage and the electrical energy it stores. Over the discharging stage, the DE is discharged, its voltage rectified and energy added to the battery. While transduction of DEs can also occur at constant voltage, it has been analytically shown that this leads to a lower amount of net energy transduced, when all other factors are held the same.

The relationship between a DEs capacitance and stretch can be characterized as follows. If the length and width of the elastomer film each increase by a factor of $\lambda$ when stretched, the area will have increased by a factor of $\lambda^{2}$. As an incompressible material, the volume must stay constant to cause a decrease in thickness by a factor of $\lambda^{2}$, leading to an increase in capacitance by a factor of $\lambda^{4}$ (equation (1b)). The reason behind the high energy density of DEs is underscored by equations (1b) thru (1d), where $\Delta E_{D E}$ is the net energy output of the DE. If it fully relaxes, the electrical energy in the film will have increased to a factor of $\lambda^{4}$ of the input electrical energy. This is in contrast to a maximum $\lambda^{2}$ factor increase in energy in conventional electrostatic transducers, a limitation of their rigid structure. 


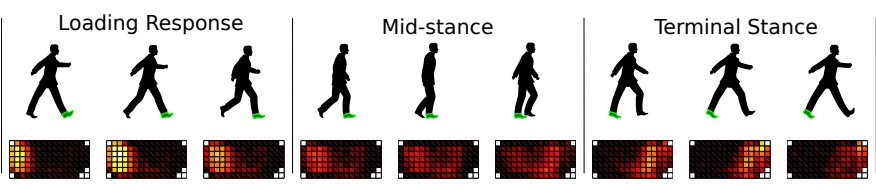

Fig. 2: Foot pressure profiles for sub-phases of stance phase.

$$
\begin{aligned}
C_{\lambda} & =\frac{\varepsilon_{0} \varepsilon_{r} \lambda^{2} A}{\frac{d}{\lambda^{2}}} \\
& =\lambda^{4} C_{0} \\
\Delta E_{D E} & =\frac{Q^{2}}{2 C_{0}}\left(\frac{1}{\lambda_{\text {final }}^{4}}-\frac{1}{\lambda_{\text {init }}^{4}}\right) \\
& =\frac{C_{\lambda_{\text {init }}} V^{2}}{2}\left(\frac{\lambda_{\text {init }}^{4}}{\lambda_{\text {final }}^{4}}-1\right)
\end{aligned}
$$

\section{B. Human Gait}

Gait is defined as the way in which movement is achieved by humans with their limbs, such as walking, running, hopping, etc. The gait cycle, or stride, is divided into two phases, the stance phase, when the limb is in contact with the ground, and the swing phase, when the limb is in the air for advancement. Although gait characteristics vary across people, human locomotion, but its very nature produces commonalities across people. One such commonality is that spatial pressure profile across the sole of the foot exhibits local correlation. Fig. 2 illustrates sub-phases of the stance phase along with plantar pressure profiles corresponding to those sub-phases. These profiles are averages over several strides from one of our datasets, collected with a high spatial-resolution plantar pressure monitoring system. They depict the heel at the left and toes and the right with lighter colors corresponding to higher pressure.

Three properties of plantar pressure that generalized to all our datasets may be observed from Fig. 2: (i) The pressure profiles present spatial correlation localized to small regions across the sole; (ii) The maximum pressure observed at different regions is different; and (iii) Different regions observe maximum pressure at different times. Even within a region of high correlation, adjacent locations may observe maximum pressure at different times. We use these properties of human gait to optimize both the configuration and control of the harvesting system.

\section{DE HaRVester Design}

Fig. 3 depicts the high level mechanical energy harvester design for DEs that follows from their mode of transduction as described in section III-A. Here the user's activity context and gait, as described by the spatio-temporal foot pressure profile for each stride, dictates the instantaneous input mechanical pressure to the DE. From equation (1c), the net energy output depends on $\lambda_{\text {init }}$, the stretch in the DE when charge is applied, and $\lambda_{\text {final }}$, the stretch in the DE when it is discharged. Therefore, the control of the charging and discharging stages are crucial to the amount of net energy harvested, and are respectively controlled by switches 1 and 2 in Fig. 3. The mechanical and electrical behavior of the DE control the outcome of the actuation and transduction stages, and are governed by the DE's physical configuration and material

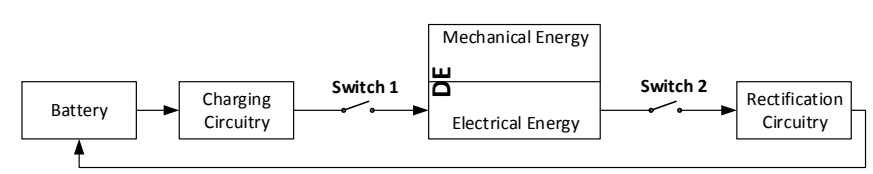

Fig. 3: Schematic of DE Energy Harvester System.

properties. Electrical losses in the DE and the rectification circuitry impact the net energy output.

In our effort to maximize the net energy harvested, we propose a physical configuration that enhances transduction efficiency. First, rather than a single DE or a few larger DE generators spanning the shoe sole, we propose an array of 99 DE micro-generators spanning the shoe sole, at locations corresponding to those in foot pressure profile diagrams in Fig. 2. As we shall see, the timing at which maximum pressure is observed at a location is crucial to control over the charging stage. Since each location may experience maximum pressure at different times, such a configuration will enable finer control of DE charging stages, thereby improving energy output. Second, the DEs harvesters will be operated in a bulge configuration that will enable direct transduction and reduce mechanical losses. Fig. 4 shows a harvester in this configuration, where DE deformation is brought about by a driver component affixed to the shoe insole. Each harvester is comprised of a circular active area, $1 \mathrm{~cm}$ in diameter, with multiple layers of DE film that are each $5 \mathrm{~mm}$ thick and prestretched to $300 \%$ by $300 \%$. The harvesters are made of the 3M manufactured VBH4905 acrylic DE [18].

\section{DE HARVESTER MODEL}

To model DE behavior analytically, we must be able to characterize the elastic forces experienced in the DE, or its stress, and therefore its geometry. In contrast to the DE transduction in Fig. 1 wherein the stretch in the DE is equal and uniform along the $\mathrm{x}$ and $\mathrm{y}$ axes, the bulge configuration involves a non-uniform stretch that is also unequal in the circumferential and longitudinal directions (Fig. 4). Although the area of the DE when stretched may be deduced via controlled measurements of its capacitance, the shape of the $\mathrm{DE}$ and therefore its stress may not be deduce in this manner. We overcome this issue by numerically solving for the shape that yields minimum area for a given vertical displacement $h$ of the DE. Such a shape will produce the minimum stretch and the minimum net energy output (Equation (1d)). The stretched DE in Fig. 4 can be deconstructed as a surface of revolution of a curve around the z-axis. In the $\mathrm{x}-\mathrm{z}$, this curve experiences boundaries at $(a, 0)$ and $(b, h)$, where $a$ and $b$ are the radii of the driver and DE active area, respectively. Discretizing the z-axis and searching for the curve that minimizes DE area for a give $h$, numerically resolves our problem. Note, that mechanical properties of the DE were exploited to prune the search space.

Given a geometric characterization of the DEs, we modeled their elastic behavior over the actuation and transduction stages via the Ogden hyper-elastic material model. DE's experience non-linear stress vs. stretch behavior and the Ogden model is commonly used to characterize this neo-hookean behavior. We experimentally derived the model's parameters with prototype DEs in the bulge configuration. Figure 5 shows the experi- 


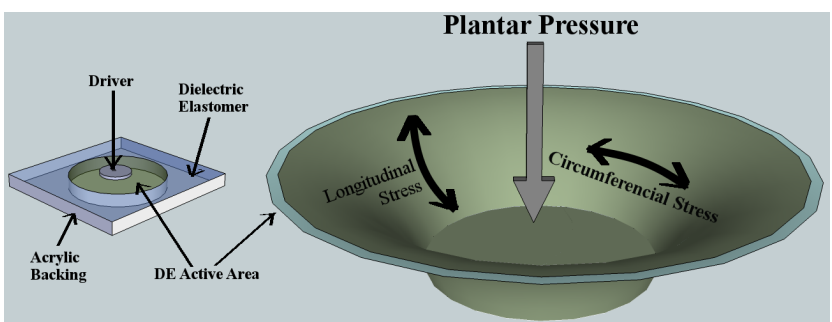

Fig. 4: DE harvester configuration.

mental setup for these stress-stretch measurements - A linear stage (Zaber linear stage model A-LSQ300A-E01) was used to move a driver affixed load cell (Transducer Techniques MDB Series) into the active area of the DE prototype that was firmly fastened in place. The displacement $h$ in the DE film was measured as the displacement in the linear stage, and the input mechanical pressure measured from the load cell together with the DE's geometric characterization were used to arrive at its stress. A non-linear least squares fit produced the parameters of the Ogden model.

$$
E_{\text {harv }}=\text { LossFact }_{\text {ckt }}\left(\Delta E_{D E}-E_{\text {loss }_{\text {elec }}}\right)
$$

The net energy harvested is proportional to the difference between the net electrical energy generated by the DE and the electrical losses of the material (equation (2)). From equation (1d), whereas $\lambda_{\text {init }}$ is arrived at with the Ogden model solely from the mechanical pressure input at the time of charging, $\lambda_{\text {final }}$ depends on the transduction stage that follows. To model DE behavior over this stage, we modified the transduction model described in [19] for the geometry involved in the bulge configuration. Here, starting from $\lambda_{\text {init }}$, the net force that leads to a change in stretch is expressed in terms of the balance between the elastic, electrostatic, gravitational and residual mechanical pressure being applied to the DE. This force balance equation is solved with a RungeKutta forth order method to produce the steady state stretch value, $\lambda_{\text {final }}$. Electrical losses modeled $E_{\text {loss }_{\text {elec }}}$ include conduction and dielectric losses in the material, and losses in the charging circuitry. The net energy harvested also considers DC-DC conversion inefficiencies in the rectification circuit (LossFact Lkt $_{\text {) }}$.

\section{DE HARVESTER ARRAY CONFIGURATION AND CONTROL OPTIMIZATION}

\section{A. Control Parameters}

Based on the description of the DE transduction mechanism, we identify 4 parameters that can be crucial to the net energy harvested.

1) Charge Timing: A key observation is that the initial stretch $\lambda_{\text {init }}$ that figures in equation (1d) is a function of the timing of the applied charge. For example, if DEs at the toes are charged at the beginning of the stance phase when they experience no input mechanical pressure, $\lambda_{\text {init }}$ will be close to 1 regardless of the user-applied pressure that follows during the stride. This will result in negative energy harvested due to the electrical losses. Therefore it is imperative that charge be applied as close to maximum stretch as possible to maximize the energy output. Since maximum stretch will be observed at maximum input mechanical pressure (due to monotonicity of the stress-stretch curve), the ability to predict when this

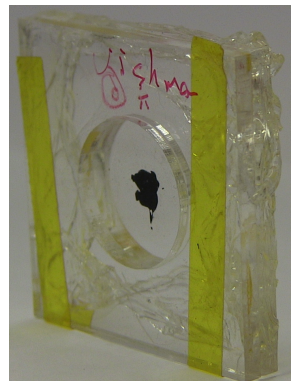

(a)

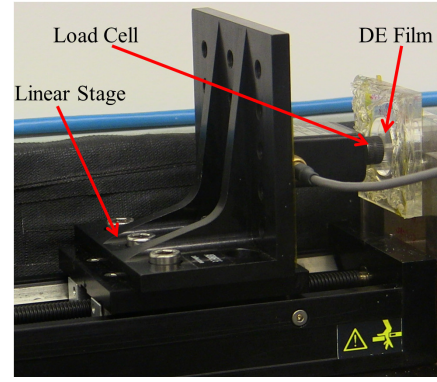

(b)
Fig. 5: (a) Prototype DE harvester, and, (b) Experimental Setup for DE prototype stress-stretch measurements.

maximum pressure will be observed is key to optimal control at the charging stage. In the following section, we will explore an adaptive control algorithm that makes use of a few foot pressure samples to enable such prediction.

2) Input Pressure: While a higher input mechanical pressure will yield a higher stretch and potentially more net energy output, this parameter is controlled by users gait over the actuation and transduction stages. However, foot-strikes yield distinct spatio-temporal properties that may be leveraged to reduce transduction in-efficiencies with an array configuration of DE micro-generators, as discussed in in section IV.

3) Harvester Thickness: A thicker DE film can withstand much more pressure without rupture, while a thinner one will stretch more at comparable applied pressures. Therefore, thinner DE films can harvest more energy at acceptable levels of applied pressure. Fig. 6 plots the stress-stretch data as well as the best fit curves for our experimental measurements for $3 \mathrm{DE}$ configurations, one with $3 \mathrm{DE}$ layers, a second with 6 layers and a third with 9 layers. It is apparent that the configuration with more layers must experience more stress to achieve similar levels of strain. Further, different regions of the foot see different levels of maximum plantar pressure (Fig. 2). To increase the energy yield of low input pressure regions of the foot, such as the arches, we propose an adaptive harvester placement strategy. Here, the thickness of a harvester in the array will depend on the amount of pressure observed over the users gait at its location. Based on the maximum pressure observed over a training dataset for each DE location, decisions will be made to fit high pressure locations with thicker harvesters and low pressure locations with thinner ones. Specifically, to address harvester lifetime and user-comfort issues, a location is occupied by the thinnest harvester for which the maximum observed pressure over the training dataset produces no more than a vertical displacement $h$ of $9 \mathrm{~mm}$, as per the stress-stretch model. This is an acceptable level of compression for soft cushioned shoes [20].

4) Applied Voltage: Equation (1d) suggests that higher applied voltages should yield higher net energy outputs, ad infinitum. However, since the applied voltage is related to the applied charge, adding too much charge to the elastomer film will create large electrostatic forces on it, which will prevent full relaxation of the film at the end of the stance phase. As $\lambda_{\text {final }}$ is driven away from 1 at higher applied voltages, extremely high voltages are undesirable. Figure 7 shows the net energy output when a harvester is exposed to 


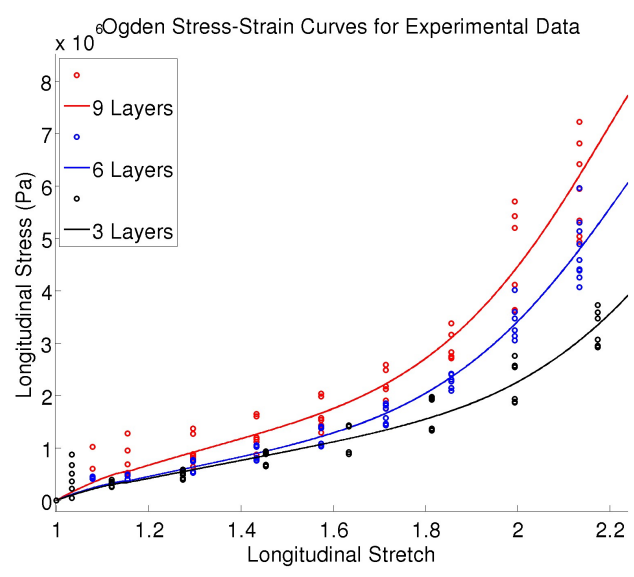

Fig. 6: Experimentally collected measurements for the stressstretch relationships of DE harvesters prototypes at different number of layers, and the corresponding best fit curves.

different input pressures and charged over a range of voltages. We observe that the output energy is not a monotonically increasing function of the applied voltage. At voltages beyond $9500 \mathrm{~V}$, and especially for input pressures at the higher end of the spectrum, the net energy output begins to decrease with increasing voltage owing to large electrostatic forces that are retained in the DE and prevent it from fully relaxing. As a result, we restrict system operation to $8000 \mathrm{~V}$. The plot also factors in the DE thickness configuration over the plantar pressure range, as described above. For this reason, the output energy does not monotonically increase with input pressure. The energy output up to $180 \mathrm{kPa}$ is governed by the thinner harvesters and they produce much more energy than the 9 layer harvester at those pressures. However, sharp transitions occur when the input pressure surpasses the user-comfort / harvester lifetime thresholds for the thinner harvester. This necessitates switching to thicker harvesters leading to sharp declines in energy output.

\section{B. Control of Harvester Array}

Aggregate net energy output is maximized if the harvesters are charged at the instant when maximum mechanical pressure is applied to them. It follows that maximum energy may be harvested over the entire array solely during the stance phase, when the foot is in contact with the ground. Hence, we focus on this phase as the temporal domain for DE operation and energy harvesting, and assume the application of one of the techniques discussed in [21] to identify the transition between the stance and swing phases with a few sensors. By detecting the transition between phases, we are able to achieve discharge stage control. In other words, all DEs may be simultaneously discharged at the end of the stance phase. However, as indicated in Fig. 2, it is not necessary that all DEs be charged at the beginning of the stance phase, if energy output is to maximized. In order to accurately predict the optimal charge timing of each harvester, we propose the use of a few pressure sensors samples. We may then leverage the local correlations in the foot pressure profiles to make accurate predictions about the timing of maximum pressure at each harvester, thereby accurately predicting the timing of maximum stretch and maximizing net energy output.

Multiple approaches may be conceptualized for the inclu-

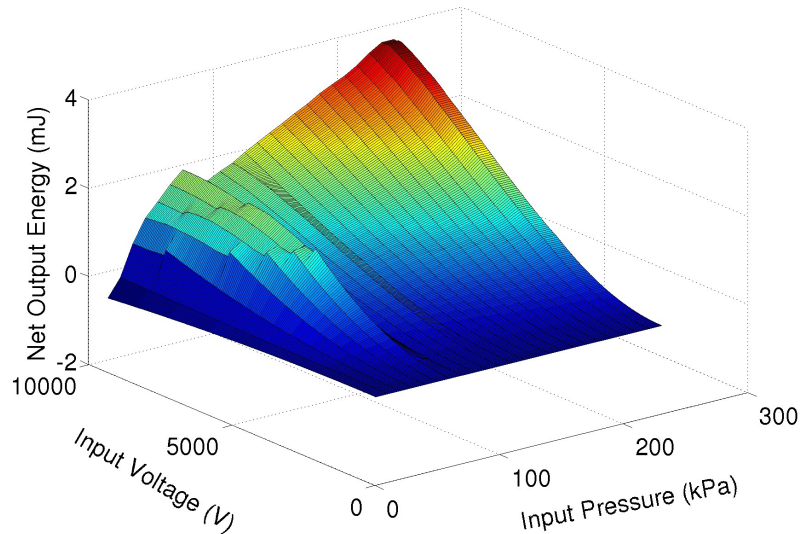

Fig. 7: Net Energy Output $\left(\Delta E_{D E}\right)$ of harvester model for different input pressures and applied voltages.

sion of foot pressure sensors into the system. The DEs may be juxtaposed with passive-resistive sensors, or, the DEs may support dual-mode operation as sensors or generators. Sensor mode operation of DEs may consume up to a few nJ more than passive-resistive sensors, but not more; Since our goal is not to maximize energy output when they are operated as sensors, DEs may be charged at a low voltage to measure a change in capacitance and calculate the applied pressure therefrom. If the DE is already charged for harvesting, the changes in its capacitance can still be measured in a similar manner.

We formally define the charge timing prediction problem as follows. We assume a maximum of $T$ epochs between the start and end of the stance phase, over which we are given the foot pressure readings at each of the harvester locations. We would like to predict the time at which maximum pressure is observed at each location in a manner that maximizes the net system energy output. Here the net system energy output is computed as the net harvester output (equation (2)) summed over all harvester, minus the energy cost of each pressure sample used in the charge timing prediction. Further, the following constraints apply:

- If the optimal charge timing for a DE is predicted as epoch $k$, the prediction must be made between epochs 1 and $k-1$.

- If the optimal charge timing for a DE is predicted as epoch $k$, the prediction must be based on samples taken solely between 1 and $k-1$.

- There is a maximum number of samples that may be used at each epoch, which may be different for different epochs. This constraint is governed by the battery power rating and the power draw of the target application that we are trying to power. However, samples taken for the purposes of the target application may also be applied to charge timing prediction.

\section{Charge Timing Prediction}

We take a statistical approach to solving the charge timing prediction problem - Given a training dataset, we apply a semi-parametric survival analysis based technique to predict optimal charge timing. Originally developed to predict machine failure times and death of biological organisms, their use 


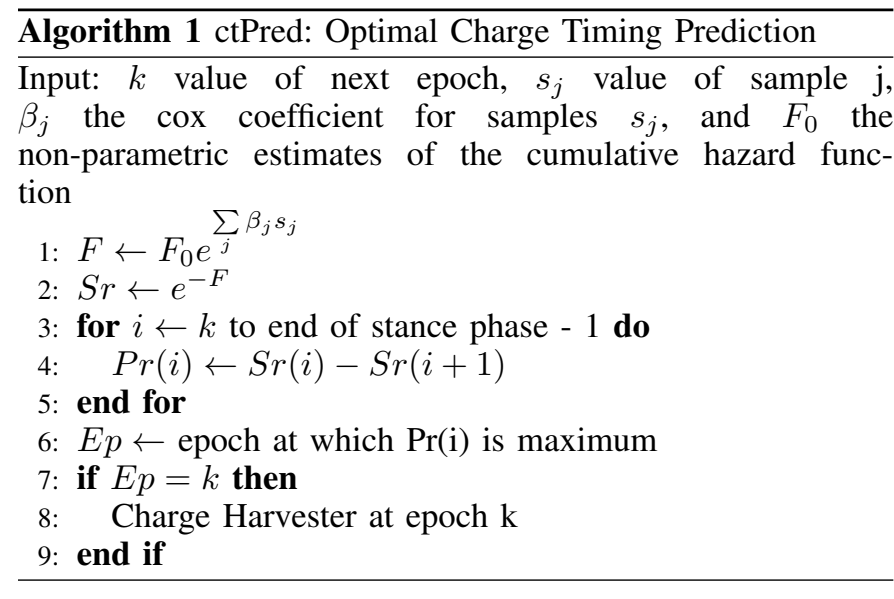

has expanded to several applications requiring time-to-event prediction. Based on a training dataset, the survival rate $S(t)$ of a harvester is the probability that its optimal charge timing hasn't yet occurred. The hazard rate $f(t)$ is the conditional probability that the optimal charge timing will occur at epoch $t$, under the condition that it hasn't occurred yet. The cumulative hazard rate $F(t)$ is the sum of the hazard rates less than or equal to $t$. And from equation ( $3 a$ ) it follows that it is the negative $\log$ of the survival rate.

$$
\begin{aligned}
f(t) & =\frac{S(t)-S(t+\Delta t)}{\Delta t S(t)} \\
F(t) & =\sum_{t^{\prime}=0}^{t} f(t) \\
& =-\log (S(t))
\end{aligned}
$$

Cox regression, $\operatorname{cox} \operatorname{Reg}()$ is a semi-parametric survival regression technique. Given a dataset of optimal charge timing for a DE over several steps, and a set of covariates $x_{1}$ thru $x_{n}$, that signify a subset of samples, the algorithm constructs a cumulative hazard rate function based on a non-parametric baseline estimate of the function, and multiplicative effects parameter composed of a linear combination of the samples $x_{i}$. The coefficients $\beta_{i}$ are derived as maximum-likelihood estimates for on the training set.

$$
F(t)=F_{0}(t) e^{\beta_{1} x_{1}+\beta_{2} x_{2}+\ldots+\beta_{n} x_{n}}
$$

Algorithm 1 outlines the procedure used to decide whether a DE should be charged at the following epoch based on the samples available so far. The algorithm is called for each uncharged harvester and at each epoch, and is provided the samples available thus far, along with the corresponding cox regression parameters. With these inputs, the algorithm computes the cumulative hazard function for the following epoch $k$ and converts the function to the corresponding survival rate function $\mathrm{Sr}$. While the survival rate function tells us the probability that the optimal charge timing won't have occurred at each future epoch, lines 3 thru 5 of the algorithm convert this to the probability that optimal charge timing will occur at each future epoch. If the highest probability co-incides with the following epoch, a decision is taken to charge the DE. The algorithm waits until just before the optimal charge timing is expected for two reasons. First, this provides additional information that is likely to lead to a better decision. Second,

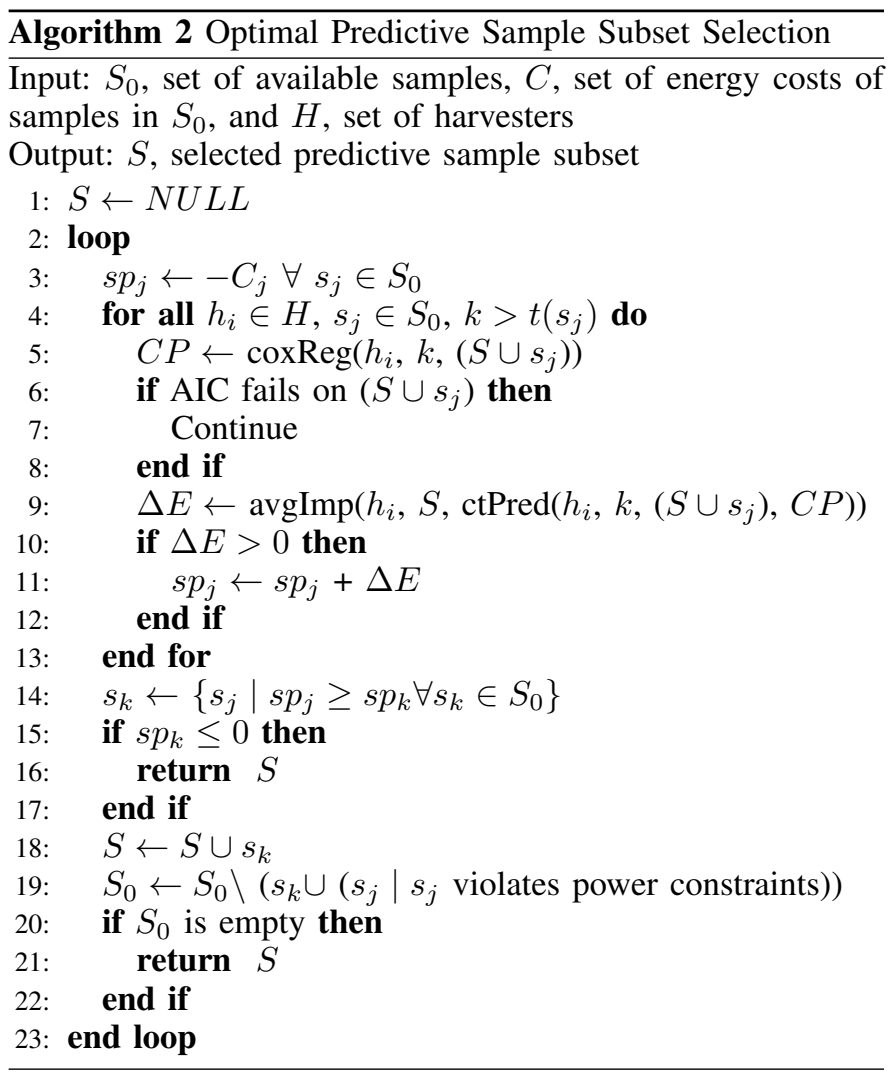

we observed that with fewer epochs remaining until the end of the stance phase, it increases the kurtosis of the optimal charge timing probability density function thereby improving our expectation.

\section{Offline Optimization}

Given our use of the survival analysis technique, the charge timing prediction problem stated in section VI-B turns into a sample selection problem that offers the best predictions while abiding by the constraints therein. We solve the problem with the stepwise-regression based optimization in algorithm 2. In the absence of samples, each harvester's timing is predicted from its baseline hazard functions. Else, each sample, $s_{j}$, is evaluated via $\operatorname{avg} \operatorname{Imp}()$ that measures the improvement $s_{j}$ affords to the prediction of harvester $h_{i}$ (line 9), averaged over training steps. This improvement contributes to $s_{j}$ 's profit $s p_{j}$ (lines 10 thru 12). The prediction's improvement is measured in terms of the increase in energy output of $h_{i}$ due to the addition of $s_{j}$ into its predictive sample subset for epoch $k$. At each iteration, the most profitable sample is selected into the predictive subset (lines 14 thru 17). Note, that if a sample is available freely, due to its necessity in the target application, its cost $C_{j}$ is 0 . Otherwise, its cost corresponds to the energy expended in acquiring the sample. Power constraints are observed by line 19, and overfitting is prevented by applying the Akaike Information Criterion (AIC) to evaluate goodness of fit before accepting a sample into a harvester's predictive subset for an epoch (lines 6 thru 8). For the sake of brevity, we have implicitly assumed that lines 11 and 18 include mechanisms to denote which harvester and epoch each included sample will be used to predict. 


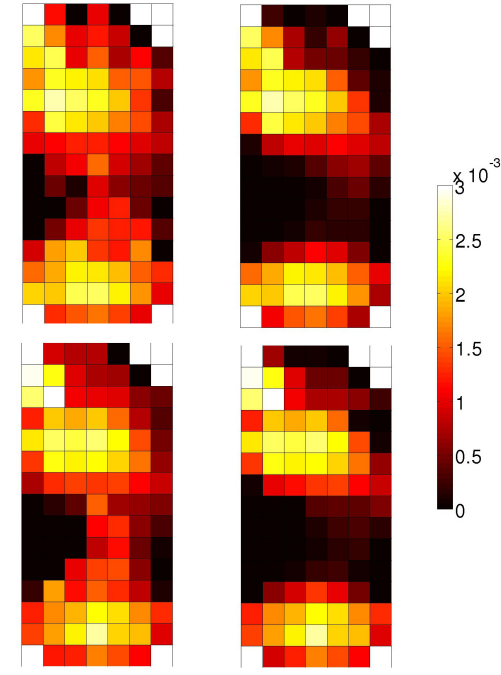

Fig. 8: The maximum energy harvestable from the left and right feet of a user in the plantar pressure dataset, averaged over strides, with uniformly thick DEs across the array (right), and those with location sensitive DE thickness (left)

\section{PERFORMANCE EVALUATION}

We evaluate the system performance with experimentally collected foot plantar pressure datasets by Hermes [10] from 3 users. Hermes is a wireless low-power human balance monitoring system, which measures foot plantar pressure via a multi-sensory array comprised of ninety-nine passive resistive pressure sensors. While Hermes was used to collect the datasets we use in our evaluation, the goal of our evaluation is, in part, to assess whether the designed system can be applied to power Hermes in a self-sustaining manner as it monitor's the wearers gait. Two of the datasets correspond to the gaits of lighter individuals, one male and one female. The third dataset corresponds to a heavier male individual. Each of the datasets offer several steps worth of data at each of the ninety-nine harvester locations. This allows us to derive performance at all harvesters and apply the predictive sample subset selection and control algorithms, thereby evaluating the system net energy output. We divide each dataset into a training subset comprised of $80 \%$ of the data and a testing subset comprised of the rest.

Figure 8 presents the maximum energy scavengable (with perfect charge timing prediction), averaged over the strides in the datasets, for both feet of a single user. Here, the maximum achievable output with and without the locationspecific thickness configuration are juxtaposed. While the areas that normally produce large amounts of plantar pressure produce exactly the same amounts of energy, surrounding areas, that were previously anemic in their output begin to look more promising. To test the predictive sample subset selection algorithm, we leveraged the research in [21] towards powerconstrained sub-sampling with Hermes. The authors therein, proposed a semantic accuracy preserving sampling strategy that reduced the energy consumption of Hermes from $72 \mathrm{~mJ}$ to $43 \mathrm{~mJ}$ per stride, under power constraints of at most 5 sensors sampled at a time. We leverage the output of the algorithm proposed in that work and constrained our algorithm by those samples. Additional samples were allowed to be chosen. if a power constraints of 5 samples per epoch permitted this.

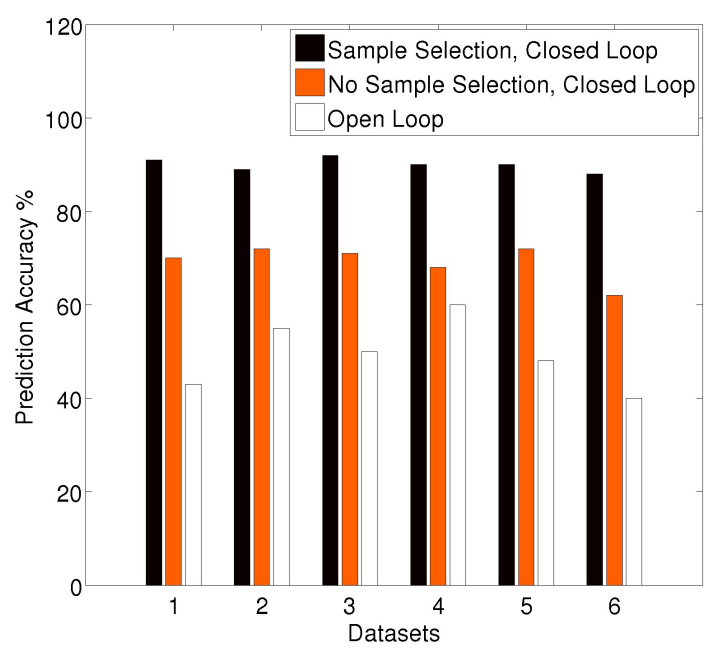

Fig. 9: Bar Plots for the prediction accuracy of different sample selection and optimal charge timing prediction techniques over the testing subsets of each of our datasets

Table I compares the average aggregate energy output of our proposed system, under both DE configurations (with and without location-specific thickness), for each of the datasets. Owing to the superior material properties of DEs and the accuracy of our algorithm, our system outperforms the piezoelectric-driven system in [1] that produced $20 \mathrm{~mJ}$ per stride. Also, across our datasets and with both our configurations, we are able to satisfy the energy demands of our target applications $(43 \mathrm{~mJ})$. Furthermore, owing to our algorithm's inbuilt penalties for overfitting, we observed minimal difference between performance over the training and testing subsets for all datasets $(<1 \%)$.

We also tested the necessity for a sampling subset selection algorithm. In figure 9, we compare the performance over the testing subset of 3 sampling subset selection techniques: (i) Our proposed technique, (ii) an adaptive system where all available samples are provided to the cox-regression algorithm, and, (iii) an algorithm that estimates charge timing of each harvester as the median epoch, over the training dataset, at which its net energy output was maximum. The comparison is shown for all 6 datasets. It is clear that an adaptive system can yield more energy. Furthermore, the prediction accuracy of the second technique over the training subset was more than $98 \%$ for each of the datasets. However, the accuracy over testing does not rise beyond $75 \%$, clearly a case of overfitting.

Another question we sought to answer was whether there

TABLE I: Performance Comparison of Foot Strike Energy Harvesting Output.

\begin{tabular}{|c|c|c|}
\hline $\begin{array}{c}\text { Scavenger } \\
\text { Mechanism }\end{array}$ & $\begin{array}{c}\text { Net Energy } \\
\text { Output (mJ) } \\
\text { - Layers }\end{array}$ & $\begin{array}{c}\text { Net Energy } \\
\text { Output (mJ) } \\
\text { - Uniform }\end{array}$ \\
\hline User 1, Left & 107.1 & 91.5 \\
User 1, Right & 120.5 & 98.3 \\
User 2, Left & 86.9 & 68.0 \\
User 1, Right & 81.9 & 69.5 \\
User 3, Left & 90.0 & 71.8 \\
User 3, Right & 80.8 & 71.2 \\
\hline
\end{tabular}




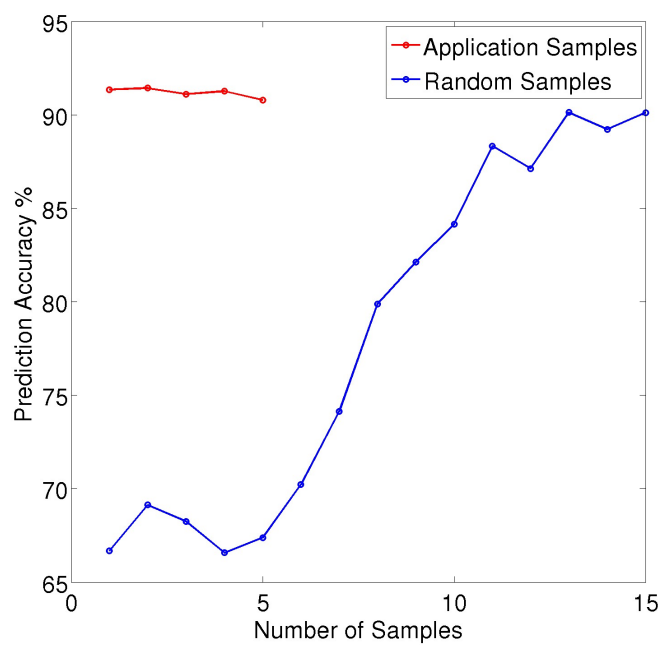

Fig. 10: Performance of our propose predictive sample subset selection algorithm under different power constraints

was something inherent in the samples afforded by the algorithm in [21] that yielded such high outputs. As it turns out, the answer is yes. Figure 10 shows the prediction accuracy from using between 1 and 5 samples per epoch restricted to the samples produced by [21]. It also shows the prediction accuracy when the samples are randomly chosen at each epoch. Note that the sample subset selection algorithm was run on the chosen samples for all cases. Both curves correspond to averages across 3 of our datasets. The blue curve in figure 10 shows that a few random samples per epoch are insufficient in producing accurate predictions. In these cases, the prediction accuracy depends mostly on the baseline hazard function as no samples are chosen into the predictive subset. As the number of samples per epoch increases beyond 22, the results improve to recognizable levels!

\section{CONCLUSION}

We have experimentally characterized and modeled a novel high energy-density harvesting technology to harvest foot-strike energy and drive low-power wearable and mobile systems. We laid out harvester configuration and control techniques to leverage characteristics of the user's gait and optimize energy output. Towards optimized control, we have proposed a novel and adaptive charge-timing prediction technique and a predictive sample selection algorithm to aid in the adaptive control of the harvesters. Finally, we have validate the performance of our algorithms over experimentally collected datasets of foot pressure produced by different users.

\section{REFERENCES}

[1] J. A. Paradiso and T. Starner, "Energy scavenging for mobile and wireless electronics," IEEE Pervasive Computing, vol. 4, no. 1, pp. 1827, Jan. 2005.

[2] I. Beretta, F. Rincon, N. Khaled, P. Grassi, V. Rana, D. Atienza, and D. Sciuto, "Model-based design for wireless body sensor network nodes," in Proc. 13th Latin American Test Workshop (LATW), 2012, pp. $1-6$.

[3] M. Vallejo, J. Recas, and J. L. Ayala, "Channel analysis and dynamic adaptation for energy-efficient wbsns," in Proc. 6th International Conference on Ubiquitous Computing and Ambient Intelligence. SpringerVerlag, 2012, pp. 42-49.
[4] H. Mamaghanian, N. Khaled, D. Atienza, and P. Vandergheynst, "Compressed sensing for real-time energy-efficient ecg compression on wireless body sensor nodes," IEEE Transactions on Biomedical Engineering, vol. 58, no. 9, pp. 2456-2466, 2011.

[5] P. Zappi, D. Roggen, E. Farella, G. Tröster, and L. Benini, "Networklevel power-performance trade-off in wearable activity recognition: A dynamic sensor selection approach," ACM Trans. Embed. Comput. Syst., vol. 11, no. 3, pp. 68:1-68:30, Sep. 2012.

[6] D. Porcarelli, D. Brunelli, M. Magno, and L. Benini, "A multi-harvester architecture with hybrid storage devices and smart capabilities for low power systems," in Proc. Intl. Symp. on Power Electronics, Electrical Drives, Automation and Motion (SPEEDAM), 2012, pp. 946-951.

[7] J. F. Antaki, G. E. Bertocci, E. C. Green, A. Nadeem, T. Rintoul, R. L. Kormos, and B. P. Griffith, "A gait-powered autologous battery charging system for artificial organs." Journal of American Society for Artificial Internal Organs, vol. 41, no. 3, 1995.

[8] N. S. Shenck and J. A. Paradiso, "Energy scavenging with shoemounted piezoelectrics," IEEE Micro, vol. 21, no. 3, pp. 30-42, May 2001.

[9] R. D. Kornbluh, R. Pelrine, H. Prahlad, A. Wong-Foy, B. McCoy, S. Kim, J. Eckerle, and T. Low, "From boots to buoys: promises and challenges of dielectric elastomer energy harvesting," Polymer, vol. 7976, no. 1, pp. 797 605-797605-19, 2011.

[10] H. Noshadi, S. Ahmadian, H. Hagopian, J. Woodbridge, F. Dabiri, N. Amini, M. Sarrafzadeh, and N. Terrafranca, "HERMES - mobile balance and instability assessment system," in Proc. BIOSIGNALS, 2010, pp. 264-270.

[11] T. Choudhury, G. Borriello, S. Consolvo, D. Haehnel, B. Harrison, B. Hemingway, J. Hightower, P. Klasnja, K. Koscher, A. Lamarca, J. Lester, J. Landay, L. Legrand, A. Rahimi, A. Rea, and D. Wyatt, "The Mobile Sensing Platform: An Embedded System for Capturing and Recognizing Human Activities," IEEE Pervasive Computing, vol. 7, no. 2, pp. 32-41, 2008.

[12] E. Costanza, S. A. Inverso, E. Pavlov, R. Allen, and P. Maes, "eye-q: Eyeglass peripheral display for subtle intimate notifications," in In Proc. Mobile HCI, year $=2006$.

[13] P. Mitcheson, E. Yeatman, G. Rao, A. Holmes, and T. Green, "Energy harvesting from human and machine motion for wireless electronic devices," Proceedings of the IEEE, vol. 96, no. 9, pp. $1457-1486$, sept. 2008.

[14] M. Hanson, H. Powell, A. Barth, K. Ringgenberg, B. Calhoun, J. Aylor, and J. Lach, "Body area sensor networks: Challenges and opportunities," Computer, vol. 42, no. 1, pp. 58 -65, jan. 2009.

[15] J. Paradiso, "Systems for human-powered mobile computing," in Proc. 43rd ACM/IEEE Design Automation Conference, 2006, pp. 645 -650.

[16] T. McKay, B. OBrien, E. Calius, and I. Anderson, "Self-priming dielectric elastomer generators," Smart Materials and Structures, vol. 19, no. 5, p. 055025, 2010.

[17] T. A. Gisby, E. P. Calius, S. Xie, and I. A. Anderson, "An adaptive control method for dielectric elastomer devices," in Proc. SPIE Electroactive Polymer Actuators and Devices, 2008, pp. 69271C-69271C8.

[18] “3M VHB TM tapes technical data sheet," $3 \mathrm{M}$, St. Paul, MN.

[19] C. Jean-Mistral, S. Basrour, and J.-J. Chaillout, "Modelling of dielectric polymers for energy scavenging applications," Smart Materials and Structures, vol. 19, no. 10, p. 105006, 2010.

[20] Optimal performance index system. http://www.opistest.com/. [Online]. Available: http://www.opistest.com/

[21] V. Goudar and M. Potkonjak, "Power constrained sensor sample selection for improved form factor and lifetime in localized bans," in Proc. 3rd Conf. on Wireless Health. New York, NY, USA: ACM, 2012.

[22] V. Goudar, Z. Ren, P. Brochu, M. Potkonjak, and Q. Pei, "Driving low-power wearable systems with an adaptively-controlled foot-strike scavenging platform," in 17th International Symposium on Wearable Computers (ISWC), 2013.

[23] V. Goudar and M. Potkonjak, "Energy-efficient sampling schedules for body area networks," in Proc. IEEE Sensors, 2012, pp. 1-4.

[24] J. Wendt, V. Goudar, H. Noshadi, and M. Potkonjak, "Spatiotemporal assignment of energy harvesters on a self-sustaining medical shoe," in Proc. IEEE Sensors, 2012, pp. 1-4. 